\title{
Real Stock Trading Using Soft Computing Models
}

\author{
Brent Doeksen ${ }^{1}$, Ajith Abraham ${ }^{2}$, Johnson Thomas ${ }^{1}$ and Marcin Paprzycki ${ }^{1}$ \\ ${ }^{1}$ Computer Science Department, Oklahoma State University, OK 74106, USA, \\ ${ }^{2}$ School of Computer Science and Engineering, Chung-Ang University, Korea, \\ ajith.abraham@ieee.org,jpt@okstate.edu,marcin@okstate.edu
}

\begin{abstract}
The main focus of this study is to compare different performances of soft computing paradigms for predicting the direction of individuals stocks. Three different artificial intelligence techniques were used to predict the direction of both Microsoft and Intel stock prices over a period of thirteen years. We explored the performance of artificial neural networks trained using backpropagation and conjugate gradient algorithm and a Mamdani and Takagi Sugeno Fuzzy inference system learned using neural learning and genetic algorithm. Once all the different models were built the last part of the experiment was to determine how much profit can be made using these methods versus a simple buy and hold technique.
\end{abstract}

\section{Introduction}

The ability to predict the direction of the stock prices is the most important factor to making money using financial prediction. All the investor really needs to know is to buy if the stock is going up in value and to sell if it is decreasing in value. This paper will delve into some of the most popular soft computing techniques for stock market modeling [1]. These methods are: neural networks, fuzzy inference system, genetic algorithm, and some heuristic techniques. The most recent studies compare indexes such as the S\&P 500, NASDAQ, and the Dow Jones [2][4][5][8][10]. The experiments done in this project examine the chaotic behavior of actual company stocks that tend to be less stable and thus harder to predict. Studies have also shown that using direction as compared to prediction can generate higher profits [4], and this study will try and capitalize on that idea. Also the prediction will examine a more realistic situation where an investor has the choice between multiple stocks, in this case 2, and chooses the stock that is mostly likely to increase in value. The experiments also compare many hybrid AI techniques and their abilities to predict a categorical output. The data for this research comprised of prices for Microsoft and Intel Corporations from January 2nd, 1990 until August $5^{\text {th }}$, 2003. Information contained for each daily report is the opening price, closing price, low price, high prices, and the volume of shares traded. Economic indicators such as the current Prime rate, Michigan's Consumer Sentiment Index, and the United States Consumer Confidences were also used to aid the models during the training phase. We explored the performance of artificial neural networks trained using backpropagation and conjugate gradient algorithm and Mamdani and Takagi Sugeno Fuzzy inference system learned using neural learning and genetic algorithm for directional prediction.

\section{Related Research}

Many papers have dealt with input selection when it comes to mapping financial indexes and stocks $[2][5][16][18][19]$. Inputs have been broken into two different types of inputs, financial and political (which tend to be qualitative). Kuo et al. [7] uses a genetic algorithm base fuzzy neural network to measure the qualitative effects on the stock price. Variable selection is critical to the success of any network for the financial viability of a company. Quah et al. [9] identified 5 key parts namely yield, liquidity, risk, growth, and momentum factors. Macroeconomic factors such as inflation and short-term interest rate [5] have to shown to have direct impacts on the stock returns. A better measure of fitness, which considers profit [10] has been suggested to replace a root means squared error. Yao and Poh [12] showed an example where a model with a low Normalized Mean Square Error (NMSE) had a lower return than a model with a higher NMSE. Brownstone [3] recommends using percentages to measure performance so that the result can be better understood by traders and other people that might need their research and are not experts in the field. Chen et al. [4] used a sliding window to predict the next day's price of the index. Everyday the network was retrained with the most recent 68 days of input with the 
attempt to predict the coming day. Commission (remuneration for services rendered) is commonly overlooked when doing research relating to stock market prediction; however, if any model is actually implemented it is going to incur fees which could greatly affect the profit predicted by the model. Chen et al. [4] considers 3 different levels of commissions and how it would affect the best buying strategy used by investors.

\section{Hurst Exponent}

Some papers have used the Hurst Exponent [10] to prove that the data is not completely random but in fact has the correspondence between the input and the output data. The Hurst Exponent can show the degree of correlation. If the exponent is 0.5 the data is completely random and no thus no network will be able to predict the output and thus it is a waste of time to attempt to learn any pattern in the data. The closer the Hurst Exponent is to one, the greater the correlation between the input and output, and a Hurst Exponent of less than 0.5 means that the input and output are indirectly proportional. It is important to note the Hurst Exponent is confined to the range of 0 to 1 .

HurstExponent $=\frac{\log (R / S)}{\log (N)}$

$\mathrm{S}$ is the standard deviation of the time series before normalization and $\mathrm{R}$ is the maximum and minimum cumulative deviations of the observation has compared with the mean of the series. $\mathrm{N}$ is the number of observations

$R_{N}=\max _{1 \leq t \leq N}\left[x_{t, N}\right]-\min _{1 \leq t \leq N}\left[x_{t, N}\right]$

$x_{t, n}$, the cumulative deviation, is describe by

$x_{t, N}=\sum_{u=1}^{t}\left(x_{u}-\mu_{N}\right)$

$\mu_{x}$ is the mean of $x_{u}$ for all $N$ elements. The Hurst exponent can be very useful in any set and allows a method of comparing sets of data.

\section{Soft Computing (SC)}

Soft computing comprises of new generation computationally intelligent hybrid systems consisting of neural networks, fuzzy inference system, approximate reasoning and derivative free optimization techniques. Neural Networks is an attempt at creating a computer that could learn in a manner similar to humans. Neural Networks can determine complex function approximations, classifications, auto associations etc. Fuzzy logic gives a set of natural language rules that are easily understood by humans. The primary advantage of fuzzy logic is its readability. For a first order TakagiSugeno model, a common rule is represented as [14]:

If $x$ is $A_{l}$ and $y$ is $B_{l}$, then $f_{l}=p_{l} x+q_{1} y+r_{l}$

where $x$ and $y$ are linguistic variables and $A_{1}$ and $B_{l}$ are corresponding fuzzy sets and $p_{l}, q_{l}, r_{l}$ are linear parameters. Usually the least squares algorithm is used to determine the linear parameters and the membership function parameters are fine tuned using a neural network learning method. Initial rules are generated using the gridpartitioning method [6]. In the inference method proposed by Mamdani the rule consequence is defined by fuzzy sets and has the following structure [13]:

if $x$ is $A_{1}$ and $y$ is $B_{1}$ then $z_{1}=C_{1}$

where $x$ and $y$ are linguistic variables, $A_{l}$ and $B_{l}$ are input fuzzy sets and $C_{l}$ the corresponding output fuzzy set. Usually a mixture of neural learning and global optimization method are used to fine tune the various rule parameters. Genetic algorithms loosely mimic the concept of natural selection. Each member is made up of a chromosome, which is normally a binary string. This chromosome defines the characteristic of the member of the population and that allows the algorithm to determine its fitness. A population is a group of members and changes from generation to generation through methods such as mutation and crossover. The fitness function is used at every generation to see which members are fit and most likely to survive to the next generation through a reproduction process.

\section{Experiment Setup and Results}

The data sets used in this paper originally had data from January 1990 to August 2003. Since the stock prices had a big variation during the entire period, all the data prior to 1997 was removed from the data set. This provided 2 services: first it reduced the size of the data set and second it gave a better split of increasing days to decreasing days. Studies have shown in classification problems it is important to have equal representations of both cases in order to prevent the network from becoming biased towards the more common value, in this case increasing days. Intel data set contained less than $1 \%$ difference in the representation of increasing days as compared with decreasing days, thus further manipulation of the data set was not required. However Microsoft data during this period had an increase in $59.6 \%$ of the days in the sample set. In order to prevent the network from heavily favoring the increased prediction, the data set had increasing predictions randomly removed until a 55/45 split was achieved. This allowed experiments to be tested to show the difference with strong bias and without a bias. Thus the last year of data from August $1^{\text {st }}, 2002$ until July $31^{\text {st }}, 2003$ was held out of set to be used as an unseen testing set in the simulation. 
All days in which the price increased or decreased by more than $10 \%$ in a single day were removed from the database as these outliers were most likely caused by external forces. Also days in which less than $0.1 \%$, or no change occurred were removed because an action performed by the investor would not affect the bottom line. The outliers for these models were determined to be days when trade volume is 4 times the average trade volume or more. For Microsoft, this value came out to any day that more than 105 million shares were traded. The prime rate [11] was also used and any day that rate was changed was removed from the data set.

Transforming the data into a network usable form is essential for the success of the network. The data in this paper was normalized using a min-max normalization. There are 16 inputs to begin with for the two data sets. The original 16 inputs that were considered are: consumer confidence index, the prime rate, Michigan consumer sentiment Index, price -1 (yesterday), price -2 (day before yesterday), price -3 , price -4 , price -5 , volume -1 (volume yesterday), low -1 , high -1 , open -1 , low -2 , high -2 , open -1 , and volume -2 . Several models were used to determine the least important inputs and then these inputs were removed before a more accurate and iterative approach could be used to determine the final inputs. A genetic algorithm with a population size of 50 was trained for 100 generations and the sensitivity about the mean test was conducted to see how important each input is relative to a particular neural network for prediction of the outputs. The result for Microsoft data set is shown below in Figure 1.

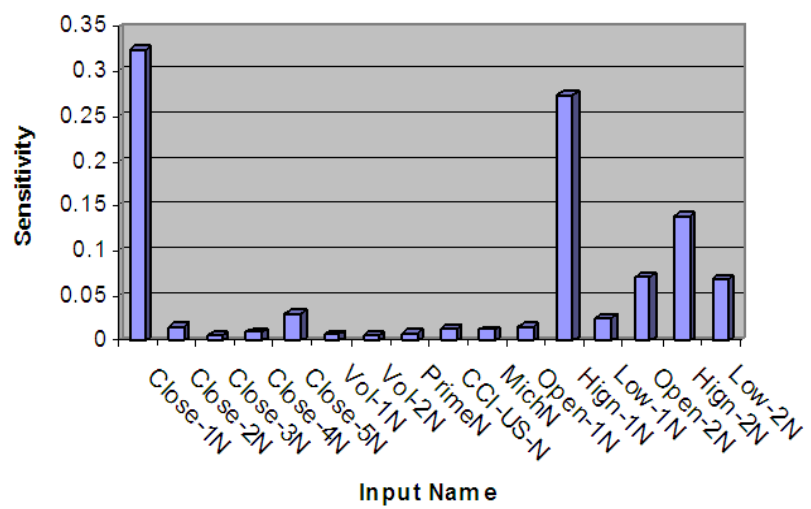

Figure. 1. Sensitivity test for Microsoft data

The sensitivity test helped to select the best 9 inputs. The number of inputs was further reduced to a much more manageable size using a greedy systematic test using a neural network classifier. Using the 9 inputs, a feed forward neural network was built and tested by removing one input randomly. The network which did the best on test was kept, thus after the first iteration there were 8 inputs left. All networks were trained 3 times with randomly initialized weights and the best network (selection of input variables) was chosen. The neural network used 20 and 7 neurons in the hidden layers. The conjugate gradient learning algorithm was used for 10,000 epochs for all the experiments. Table 1 shows the results that determined CCI (Consumer Confidence Index) should be removed from Microsoft data. The network with CCI input had a NMSE of 0.007506 , which was worse than the network that had the input removed $(0.007119)$. Table 2 illustrates the next iteration, which elects volume to be removed with a NMSE of 0.006871 .

Table 1. Snapshot of input reduction with 8 variables

\begin{tabular}{|c|c|r|}
\hline $\begin{array}{c}\text { Input } \\
\text { variable }\end{array}$ & $\begin{array}{c}\text { MSE cross } \\
\text { validation }\end{array}$ & NMSE on testing \\
\hline CCI & 0.000520791 & $\mathbf{0 . 0 0 7 1 1 8 9 0 8}$ \\
\hline close-3 & 0.000535407 & 0.007293936 \\
\hline volume & 0.000527196 & 0.007308423 \\
\hline high-2 & 0.000514665 & 0.007397344 \\
\hline close-2 & 0.000529705 & 0.007571371 \\
\hline open-1 & 0.000515599 & 0.007645692 \\
\hline high-1 & $\mathbf{0 . 0 0 0 5 1 2 6 5 5}$ & 0.007789516 \\
\hline close-1 & 0.000600180 & 0.008804343 \\
\hline
\end{tabular}

Table 2. Snapshot of input reduction with 7 variables

\begin{tabular}{|c|r|r|}
\hline $\begin{array}{c}\text { Input } \\
\text { variable }\end{array}$ & $\begin{array}{c}\text { MSE cross } \\
\text { validation }\end{array}$ & NMSE on testing \\
\hline volume-1 & 0.000514894 & $\mathbf{0 . 0 0 6 8 7 1 0 9 4}$ \\
\hline close-2 & 0.000517851 & 0.007191489 \\
\hline high-2 & $\mathbf{0 . 0 0 0 5 0 9 0 8 2}$ & 0.007259947 \\
\hline close-3 & 0.000519643 & 0.007460574 \\
\hline high-1 & 0.000532197 & 0.007658685 \\
\hline open-1 & 0.000524406 & 0.007685916 \\
\hline close-1 & 0.000583583 & 0.008093149 \\
\hline
\end{tabular}

This process was iterated until the dataset contained the 5 most significant inputs. The final selection of inputs is: close-1, open-1, high-1, high-2, and close-3.

A soft computing paradigm could be used to model a regression or classification task. When performing regression, all the 5 inputs are given to the network, and the output is the stock price for the next day. Once a test is completed the predicted price is compared with yesterday's price to see if the price increased or decreased. Classification is much more straightforward. Before presenting the data to the network the output is translated to 1 , for increase, or 0 for decrease. And the objective of the network is to correctly predicted 0 or 1 . Some studies have shown that classification can out perform their regression counterparts. Table 3 illustrates the regression/classification modeling results using neural networks. 
Table 3. Regression/classification performance

\begin{tabular}{|c|c|c|c|c|c|}
\hline \multirow{2}{*}{$\begin{array}{c}\text { Neurons } \\
\text { N }\end{array}$} & MSE & NMSE & Correct & Decrease & Increase \\
\cline { 3 - 6 } & \multicolumn{5}{|c|}{ Regression } \\
\hline 28 & 0.00047 & 0.00666 & 57.94 & 37.50 & 78.14 \\
\hline 42 & $\mathbf{0 . 0 0 0 4 6}$ & 0.00679 & 52.71 & 31.65 & 73.52 \\
\hline 26 & 0.00049 & 0.00734 & 54.45 & 36.33 & 72.36 \\
\hline \multicolumn{6}{|c|}{ Classification } \\
\hline 24 & 0.3702 & NA & 59.98 & 37.50 & 82.19 \\
\hline 48 & $\mathbf{0 . 3 6 8 9}$ & NA & 59.40 & 35.16 & $\mathbf{8 3 . 3 5}$ \\
\hline 22 & 0.3745 & NA & $\mathbf{6 2 . 5 9}$ & $\mathbf{4 2 . 1 8}$ & 82.77 \\
\hline
\end{tabular}

When dealing with random sets, showing the same tests for different randomly selected data sets shows the results are realizable. The Microsoft data set used in the previous 2 sections was randomized 3 different times and networks were built for each data set using the same standards discussed in previous sections to ensure the best possible networks were created. Results for the three random data sets are illustrated in Figure 2 and Table 4. As evident, the performance is comparable and thus the results can be reliable for any random grouping of the dataset.

The test standards listed below were used for all the models unless otherwise noted on the results. All neural network models were trained for 10,000 epochs and cross validation was used to terminate training after 200 epochs with no improvement. Also all networks were trained 3 times each with randomized starting weights to ensure the best possible network by minimizing the chance of obtaining a local minima. The number of hidden neurons in the first layer would vary from 10 to 50 , with a step size of 2 , to determine which network was best at learning the data. When a 2-layered neural network was designed the second layer would contain the ceiling of the log of the number of neurons in the first layer. The training set was broken up as $80 \%$ training and $20 \%$ cross validation. Table 5 reveals the performance of backpropagation and conjugate gradient algorithm for the directional prediction of Microsoft stocks for different number of hidden neurons.

Performance of the Mamdani Fuzzy Inference System (FIS) is illustrated in Table 6. Three Membership Functions (MF's) were allocated to each input variable and the membership function parameters were fine tuned using 200 epochs of gradient descent algorithm and 50 generations of Genetic Algorithm (GA). Uniform crossover and a population size of 50 was used for the GA. Similarly the performance of Takagi Sugeno fuzzy inference system is illustrated in Table 7 for the different number of Membership Functions (MF's)/input variable for 300 epochs of gradient descent and least squares method.

Table 4. Performance for the different random data sets

\begin{tabular}{|c|c|c|c|c|}
\hline \multicolumn{5}{|c|}{ Classification Using Difference Sets (Microsoft) } \\
\hline $\begin{array}{c}\# \\
\text { Neurons }\end{array}$ & MSE & Correct & Decrease & Increase \\
\cline { 2 - 5 } Set 1 & \\
\hline 22 & 0.3745 & $\mathbf{6 2 . 5 9 \%}$ & $\mathbf{4 2 . 1 8 \%}$ & $82.77 \%$ \\
\hline 24 & 0.3702 & $59.98 \%$ & $37.50 \%$ & $82.19 \%$ \\
\hline 48 & $\mathbf{5 . 3 6 8 9}$ & $59.40 \%$ & $35.16 \%$ & $83.35 \%$ \\
\hline \multicolumn{5}{|c|}{ Set 2 } \\
\hline 34 & 0.3920 & $55.91 \%$ & $16.21 \%$ & $90.03 \%$ \\
\hline 20 & 0.3924 & $58.23 \%$ & $10.40 \%$ & $\mathbf{9 5 . 9 2 \%}$ \\
\hline 38 & 0.3905 & $59.40 \%$ & $26.27 \%$ & $87.86 \%$ \\
\hline \multicolumn{5}{|c|}{ Set 2 } \\
\hline 42 & 0.379365 & $60.85 \%$ & $36.33 \%$ & $80.49 \%$ \\
\hline 32 & 0.382199 & $59.98 \%$ & $36.99 \%$ & $78.39 \%$ \\
\hline 54 & 0.380529 & $60.85 \%$ & $37.64 \%$ & $79.44 \%$ \\
\hline
\end{tabular}

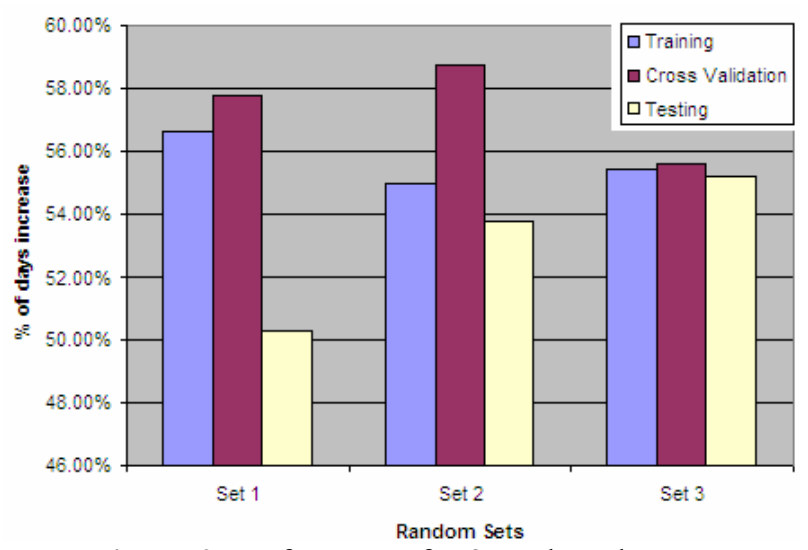

Figure 2. Performance for 3 random data sets

Table 5. Performance of neural networks

\begin{tabular}{|c|c|c|c|c|}
\hline $\begin{array}{c}\text { Hidden } \\
\text { Neurons }\end{array}$ & MSE & $\begin{array}{c}\text { Overall } \\
\text { Correct }\end{array}$ & Decrease & Increase \\
\hline & \multicolumn{5}{|c|}{ Backpropagation } \\
\hline 40 & 0.3748 & $59.10 \%$ & $41.60 \%$ & $76.41 \%$ \\
\hline 36 & 0.3702 & $\mathbf{6 1 . 1 4 \%}$ & $36.92 \%$ & $\mathbf{8 5 . 0 8 \%}$ \\
\hline & \multicolumn{5}{|c|}{ Conjugate Gradient } \\
\hline 48 & $\mathbf{0 . 3 6 8 9}$ & $59.40 \%$ & $35.16 \%$ & $83.35 \%$ \\
\hline 22 & 0.3745 & $\mathbf{6 2 . 5 9 \%}$ & $\mathbf{4 2 . 1 8 \%}$ & $82.77 \%$ \\
\hline
\end{tabular}

Table 6. Performance of Mamdani FIS

\begin{tabular}{|c|c|c|c|}
\hline Epochs & $\begin{array}{c}\text { RMSE } \\
\text { Training }\end{array}$ & NMSE Testing & $\begin{array}{c}\text { \% } \\
\text { Correct }\end{array}$ \\
\hline 1000 & 0.0232 & 0.0414 & $\mathbf{5 3 . 3 1}$ \\
\hline 2000 & $\mathbf{0 . 0 2 3 0}$ & 0.0412 & 53.01 \\
\hline
\end{tabular}

Table 7. Performance of Takagi-Sugeno FIS

\begin{tabular}{|l|l|l|}
\hline \# MF's & NMSE on testing & $\%$ Correct \\
\hline
\end{tabular}




\begin{tabular}{|l|l|l|}
\hline 3 & $\mathbf{0 . 0 0 3 8 1 1}$ & 53.67 \\
\hline 4 & 0.004515 & $\mathbf{5 6 . 0 0}$ \\
\hline 5 & 0.005447 & 55.33 \\
\hline
\end{tabular}

\subsection{Stock Trading Simulation}

Each soft computing model was given $\$ 100$ at the beginning of the testing period, August 1, 2003, and the model bought if it predicted an increase with over a 50\% certainty. The model would hold onto the stock until it came to a day, which had an increase certainty of less than $50 \%$, and then it would sell the stock. Table 8 shows an example of this strategy.

Table 8. Financial Simulation Model

\begin{tabular}{|c|c|c|c|}
\hline $\begin{array}{c}\text { Increase } \\
\text { Output }\end{array}$ & Action & $\begin{array}{c}\text { \# of } \\
\text { shares }\end{array}$ & Cash \\
\hline 0.460738 & Stay & 0 & $\$ 100.00$ \\
\hline 0.617069 & Buy & 4.411116 & \$ - \\
\hline 0.566166 & Hold & 4.411116 & $\$$ \\
\hline 0.551663 & Hold & 4.411116 & $\$$ \\
\hline 0.595844 & Hold & 4.411116 & $\$$ \\
\hline 0.521784 & Hold & 4.411116 & \$ - \\
\hline 0.489340 & Sell & 0 & $\$ 106.93$ \\
\hline 0.552275 & Buy & 4.483247 & \$ - \\
\hline 0.478012 & Sell & 0 & $\$ 107.69$ \\
\hline 0.651398 & Buy & 4.617822 & $\$ \quad-$ \\
\hline 0.485218 & Sell & 0 & $\$ 113.78$ \\
\hline 0.557304 & Buy & 4.612206 & $\$ \quad-$ \\
\hline 0.479946 & Sell & 0 & $\$ 114.29$ \\
\hline 0.461905 & Stay & 0 & $\$ 114.29$ \\
\hline
\end{tabular}

A similar model was also built which earned the prime rate for any money that was not invested in the stock and this resulted in only marginal improvement for all models. As depicted in Figure 3, profit was improved as much as $889 \%$ over a straightforward buy and hold strategy using the neural network models. The performance was dependant on the number of hidden neurons. The model, using 44 hidden neurons was able to profit $\$ 103.17$ in a one-year period with an initial investment of $\$ 100.00$. For comparison, if the same money bought stocks at the beginning of the period and sold the stocks at the end of the period it would have made a profit of $\$ 10.43$, this is referred to as a buy and hold strategy. This model predicted the direction of the stock correctly $63 \%$ of the time. Despite the lack luster performance the model was able to predict correctly when it counts most. The biggest draw back of such a scheme in the real world is commission. The model mentioned above bought and sold stock a total of 143 times during the 252 trading days in the simulation period. The total number of trades for Intel and Microsoft is shown in Figure 4.

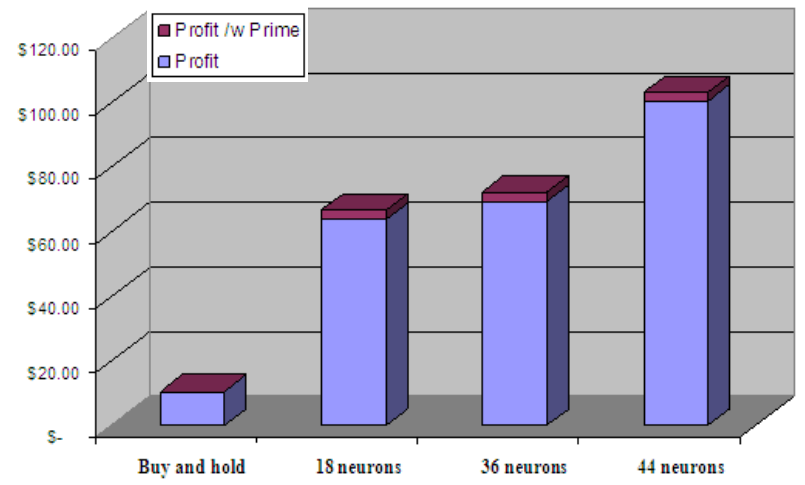

Figure 3. Stock trading simulation for Microsoft

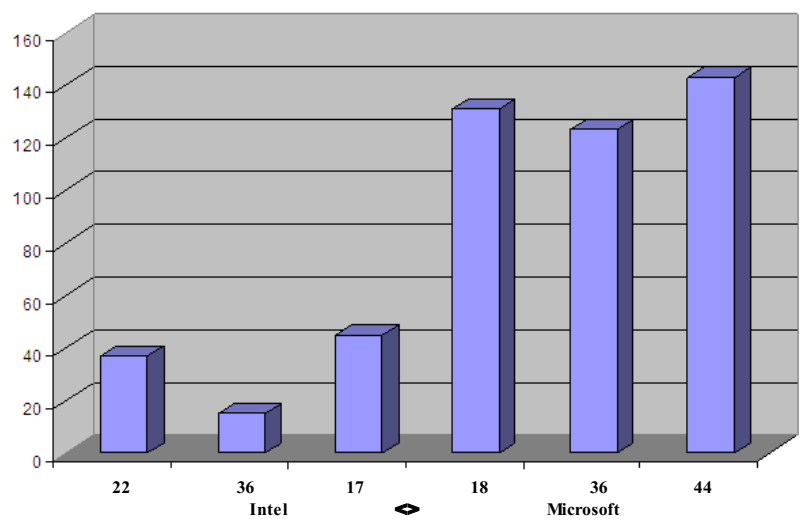

Figure 4. Volume of trades using the SC models

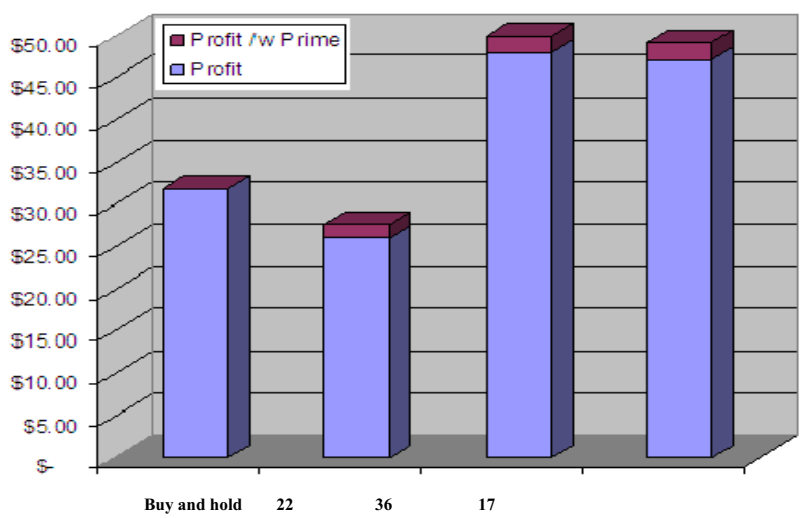

Figure 5. Stock trading simulation for Intel

Compared to Microsoft, the prediction on Intel's stock was inferior in all cases. Two of the 3 models were able to beat the buy and hold strategy. The only plus side of Intel performance is it minimized the number of transactions, thus it would incur less commission if it was actually implemented. Table 9 illustrates the best models picked by the lowest MSE on the cross validation set for both Intel and Microsoft. The profit seen by the best Model for Intel increased profit over the buy and hold strategy by $55 \%$. 
Table 9. Performance of the optimal models for trading

\begin{tabular}{|c|c|c|c|}
\hline \multicolumn{2}{|c|}{ Mean Squared Error } & \multirow{2}{*}{ Correct } & \multirow{2}{*}{ Profit (\$) } \\
\cline { 1 - 2 } Training & Cross Valid. & \\
\hline \multicolumn{3}{|c|}{ Microsoft (44 neurons) } \\
\hline 0.3959 & $\mathbf{0 . 3 9 6 8}$ & $\mathbf{6 3 . 3 2} \%$ & $\mathbf{1 0 3 . 1 7}$ \\
\hline \multicolumn{4}{|c|}{ Intel (17 neurons) } \\
\hline $\mathbf{0 . 3 9 5 7}$ & 0.3972 & $54.98 \%$ & 49.23 \\
\hline
\end{tabular}

\section{Conclusions}

The ability to predict stocks on a daily basis is a very difficult problem even for the most advance networks. The Hurst component confirmed the hypothesis, which is prediction is possible but especially difficult. Surprisingly, the Consumer Confidence Index and Prime Rate were not able to improve the predictability of these networks. It is clear from all the tests that the networks were able to learn the pattern in Microsoft's data much more easily than Intel's data, thus it might be possible that another stock is more learnable than Microsoft. Through the uses of many techniques it is possible to correctly predict the direction of the stock $63 \%$ of the time for a large company like Microsoft. Thus, this research provides the groundwork for financial trading using some of the well known soft computing paradigms. Even the worst model used for Microsoft produced a return on investment of $66 \%$, and the best network scored an astounding $103 \%$ return. This study also demonstrated that picking the correct stock is as important as building the best network; as the best network for Intel was outperformed by the worst network on Microsoft data. The biggest downfall of these networks is that the transaction cost of buying and selling stocks would be very costly. However, it would be feasible to fine tune the buy and sell strategy to lower this cost.

\section{References}

[1] Abraham A., Intelligent Systems: Architectures and Perspectives, Recent Advances in Intelligent Paradigms and Applications, Abraham A., Jain L. and Kacprzyk J. (Eds.), Studies in Fuzziness and Soft Computing, Springer Verlag Germany, Chapter 1, pp. 1-35, 2002.

[2] Abraham, A., Philip, N. S., and Saratchandran, P. "Modeling Chaotic Behavior of Stock Indices Using Intelligent Paradigms. Neural, Parallel and Scientific Computations, 11 (2003): 143-160.

[3] Brownstone, D. Using Percentage Accuracy to Measure Neural Network Predictions in Stock
Market Movements. Neurocomputing 10 (1996): 237-250.

[4] Chen, A.S., Leung, M.T., and Daouk, H. Application of Neural Networks to an Emerging Financial Market: Forecasting and Trading the Taiwan Stock Index. Computers and Operations Research 30 (2003): 901-923.

[5] Izumi, K. and Ueda, K. "Analysis of Exchange Rate Scenarios Using an Artificial Market Approach." Proceeding of the International Conference on Artificial Intelligence 2 (1999): 360-366.

[6] Jang, J.S.R. "ANFIS: Adaptive-Network-Based Fuzzy Inference System." IEEE Transactions on Systems, Man, and Cybernetics 23 (1993): 665-684.

[7] Kuo, R.J., Chen, C.H., and Hwang, Y.C. "An Intelligent Stock Trading Decision Support System through Integration of Genetic Algorithm Based Fuzzy Neural Network and Artificial Neural Network." Fuzzy Sets and Systems, 118 (2001): 21-24.

[8] O'Brian, T. V. "Neural Nets for Direct Marketers" Marketing Research, Volume 6, Issue 1

[9] Quah, T.S. and Srinivasan, B. "Improving Returns on Stock Investment through Neural Network Selection." Expert Systems with Applications 17 (1999): 295-301.

[10] Yao, J.T. and Tan, C.L. "A Study on Training Criteria for Financial Time Series Forecasting." Proceedings of International Conference on Neural Information Processing. Nov. 2001: 772-777.

[11] Prime rate: http://research.stlouisfed.org/fred2/data/PRIME.txt

[12] Yao, J. and Poh, H.L. "Forecasting the KLSE Index Using Neural Networks." IEEE International Conference on Neural Networks 2 (1995) 10121017.

[13] Mamdani E H and Assilian S, An experiment in Linguistic Synthesis with a Fuzzy Logic Controller, International Journal of Man-Machine Studies, Vol. 7, No.1, pp. 1-13, 1975.

[14] Sugeno M, Industrial Applications of Fuzzy Control, Elsevier Science Pub Co., 1985. 